\title{
Profiling of the drug resistance of thousands of Src tyrosine kinase mutants uncovers a regulatory network that couples autoinhibition to the dynamics of the catalytic domain
}

\author{
Sujata Chakraborty, ${ }^{1,7}$ Ethan Ahler, ${ }^{2,3,7}$ Jessica J. Simon, ${ }^{1}$ Linglan Fang, ${ }^{1}$ Zachary E. Potter, ${ }_{\star}^{1}$ Katherine A. \\ Sitko, ${ }^{2}$ Jason J. Stephany, ${ }^{2}$ Miklos Guttman, ${ }^{4}$ Douglas M. Fowler, ${ }^{2,5}$ and Dustin J. Maly ${ }^{1,6,8}{ }^{*}$ \\ ${ }^{1}$ Department of Chemistry, University of Washington, Seattle, WA 98195, USA, ${ }^{2}$ Department of Genome Sciences, \\ University of Washington, Seattle, WA 98195, USA, ${ }^{3}$ Molecular and Cellular Biology, University of Washington, \\ Seattle, WA 98195, USA, ${ }^{4}$ Department of Medicinal Chemistry, University of Washington, Seattle, WA 98195, USA, \\ ${ }^{5}$ Department of Bioengineering, University of Washington, Seattle, WA 98195, USA, ${ }^{6}$ Department of Biochemistry, \\ University of Washington, Seattle, WA 98195, USA, \\ ${ }^{7}$ These authors contributed equally. \\ ${ }^{8}$ Lead Contact \\ *Correspondence: djmaly@uw.edu (D.J.M.), dfowler@uw.edu (D.M.F.).
}

\section{SUMMARY}

Protein kinase inhibitors are effective cancer therapies, but acquired resistance often limits clinical efficacy. Despite the cataloguing of numerous resistance mutations with model studies and in the clinic, we still lack a comprehensive understanding of kinase inhibitor resistance. Here, we measured the resistance of thousands of Src tyrosine kinase mutants to a panel of ATP-competitive inhibitors. We found that ATP-competitive inhibitor resistance mutations are distributed throughout Src's catalytic domain. In addition to inhibitor contact residues, residues that participate in regulating Src's phosphotransferase activity were prone to the development of resistance. Unexpectedly, a resistance-prone cluster of residues that are on the top face of the N-terminal lobe of the catalytic domain contributes to Src autoinhibition by reducing the dynamics of the catalytic domain, and mutations in this cluster led to resistance by lowering inhibitor affinity and promoting kinase hyperactivation. Together, our studies demonstrate how comprehensive profiling of drug resistance can be used to understand potential resistance pathways and uncover new mechanisms of kinase regulation.

\section{INTRODUCTION}

Small molecule, ATP-competitive kinase inhibitors have revolutionized the treatment of specific cancers (Gharwan and Groninger, 2016; Zhang et al., 2009) (PMID: 34002056). Unfortunately, as with many targeted cancer therapies, the efficacy of ATP-competitive kinase inhibitors is frequently limited by the rapid development of resistance. Mechanisms of ATP-competitive kinase inhibitor resistance are often complex and heterogeneous (Garraway and Janne, 2012; Lovly and Shaw, 2014) but point mutations that render a target kinase less sensitive to inhibition are common. How do these kinase mutations result in resistance to an ATPcompetitive inhibitor? One frequently observed mechanism is through the disruption of favorable binding interactions with the inhibitor. For example, mutation of the "gatekeeper" residue, which sits near the back of the ATP-binding pocket, to a bulkier residue that restricts the size of an adjacent hydrophobic pocket is one of the major pathways of resistance observed in the clinic (Gibbons et al., 2012). Similarly, other inhibitor contact residues are also commonly observed sites of ATP-competitive inhibitor resistance (Balzano et al., 2011). However, the mechanistic basis for many clinically observed mutations that do not directly influence drug binding are less clear, and even mutations that affect inhibitor binding can confer resistance through additional mechanisms. For example, while gatekeeper residue mutations often lead to a reduction in inhibitor affinity they can also decrease inhibitor efficacy by increasing the ability of ATP to compete for ATP-binding site occupancy (Yun et al., 2008) and have been shown to lead to hyperactivation of some tyrosine kinases (Azam et al., 2008). Therefore, studies that allow the comprehensive profiling of ATP-competitive inhibitor resistance are valuable in identifying potential sites of inhibitor resistance and also providing new insight into kinase regulation and activity.

To obtain insight into different mechanisms of drug resistance, we used a deep mutational scanning (DMS) approach (Fowler et al., 2010; Fowler and Fields, 2014) to investigate the impact of nearly every mutation in Src kinase's catalytic domain on the efficacy of a panel of ATP-competitive inhibitors. Using this approach, we first established Src's pattern of resistance to the clinically-approved inhibitor dasatinib. As expected, mutations at sites within the catalytic domain that interact with dasatinib were capable of conferring resistance. We also observed that residues involved in the autoinhibition of Src's phosphotransferase activity were particularly 
prone to the development of resistance to dasatinib. Next, we profiled a matched set of conformation-selective, ATP-competitive inhibitors to compare how resistance emerges in response to different modes of ATPcompetitive inhibition. We identified a number of unique resistance mutations for each conformation-selective inhibitor and a shared set of residues that were highly susceptible to the development of resistance. Further investigation of a spatially-defined cluster of residues located on the solvent-exposed N-terminal lobe of Src's catalytic, which we refer to as the $\beta 1 / \beta 2$ resistance cluster, demonstrated that mutations at these residues were capable of reducing ATP-competitive inhibitor affinity. Biochemical analyses revealed that most mutations in the $\beta 1 / \beta 2$ resistance cluster led to hyperactivation of Src's phosphotransferase activity and that this structural element also contributes to the autoinhibited state of Src by reducing the dynamics of the catalytic domains $\mathrm{N}$ terminal lobe, which resistance mutations release. Together, our results show how a previously unrecognized component of Src's autoinhibitory regulatory network is susceptible to the development of drug resistance.

\section{RESULTS}

A yeast-based growth assay for profiling drug resistance. To comprehensively profile mechanisms of inhibitor resistance in Src, we used an assay that relies on the correlation between Src's phosphotransferase activity and its toxicity in S. cerevisiae (Ahler et al., 2019; Brugge et al., 1987; Kritzer et al., 2018). We reasoned that it would be possible to determine the drug sensitivity of individual Src variants by measuring Srcmediated yeast toxicity in the presence of various ATP-competitive inhibitors (Figure 1A). Prior to performing a parallel analysis of Src mutants, we validated that ATP-competitive inhibitors, including the highly potent Src inhibitor dasatinib (Figure 1B), were capable of dose-dependently rescuing the growth inhibition that resulted from expressing wild-type (WT), myristoylated, full-length Src (Src myr , see Table S1 for all Src constructs) in yeast (Figure S1A-D). Next, we transformed a barcoded library (Table S2) of Src ${ }^{\text {myr }}$ in which the catalytic domain was mutagenized into yeast, performed outgrowth in the presence of 25 or $100 \mu \mathrm{M}$ dasatinib and collected samples at various time points. At each time point, we extracted plasmid DNA, amplified barcodes, and then deeply sequenced the barcode amplicons to quantify the frequency of each Src variant. From these variant frequency data, we calculated activity scores for $\sim 3,500$ single amino acid Src mutants at two different dasatinib concentrations by averaging two biological replicates (Figure 1C-1F, S1E-F).

For each concentration of dasatinib treatment, we defined a Src mutant as being drug resistant if it showed an activity score greater than the two times the standard deviation of the distribution of synonymous mutants (Figure 1D, 1E). We found that patterns of dasatinib resistance looked similar for both dasatinib concentrations but with a narrower distribution of activity scores for $100 \mu \mathrm{M}$ dasatinib treatment (Figure 1D, 1E). Therefore, we further analyzed the data obtained from $25 \mu \mathrm{M}$ dasatinib treatment, where a wider spectrum of mutational effects on resistance were apparent. 
A
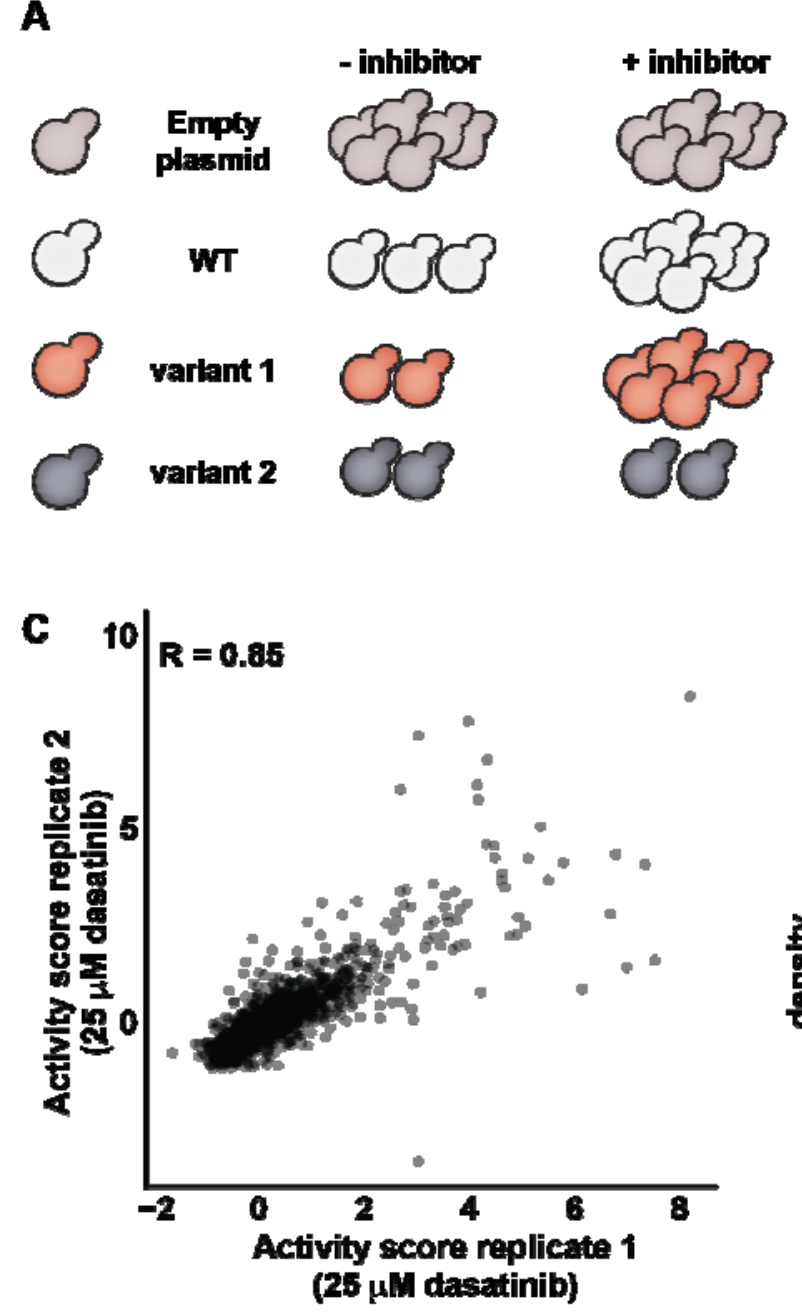

$\mathbf{E}$

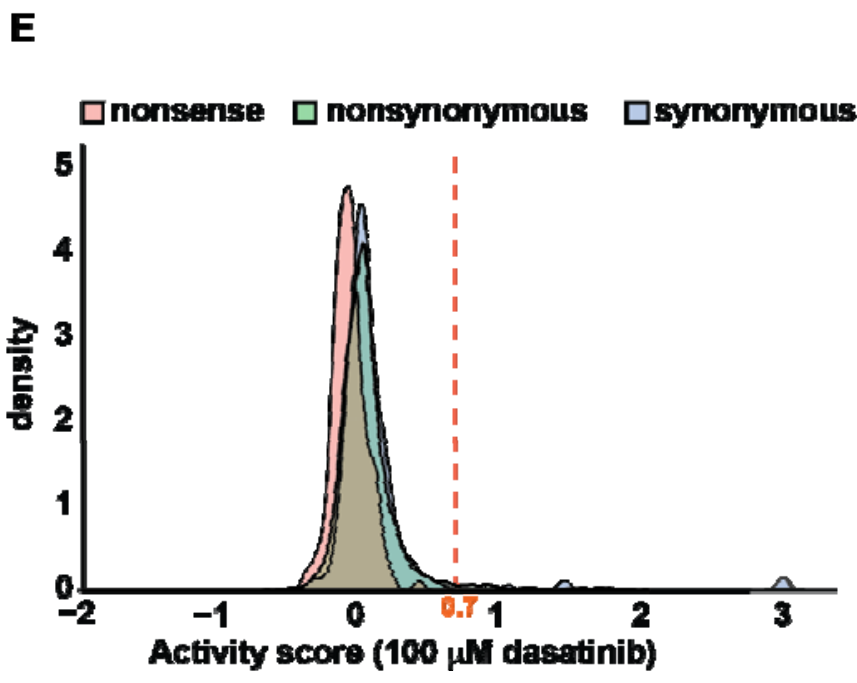

D
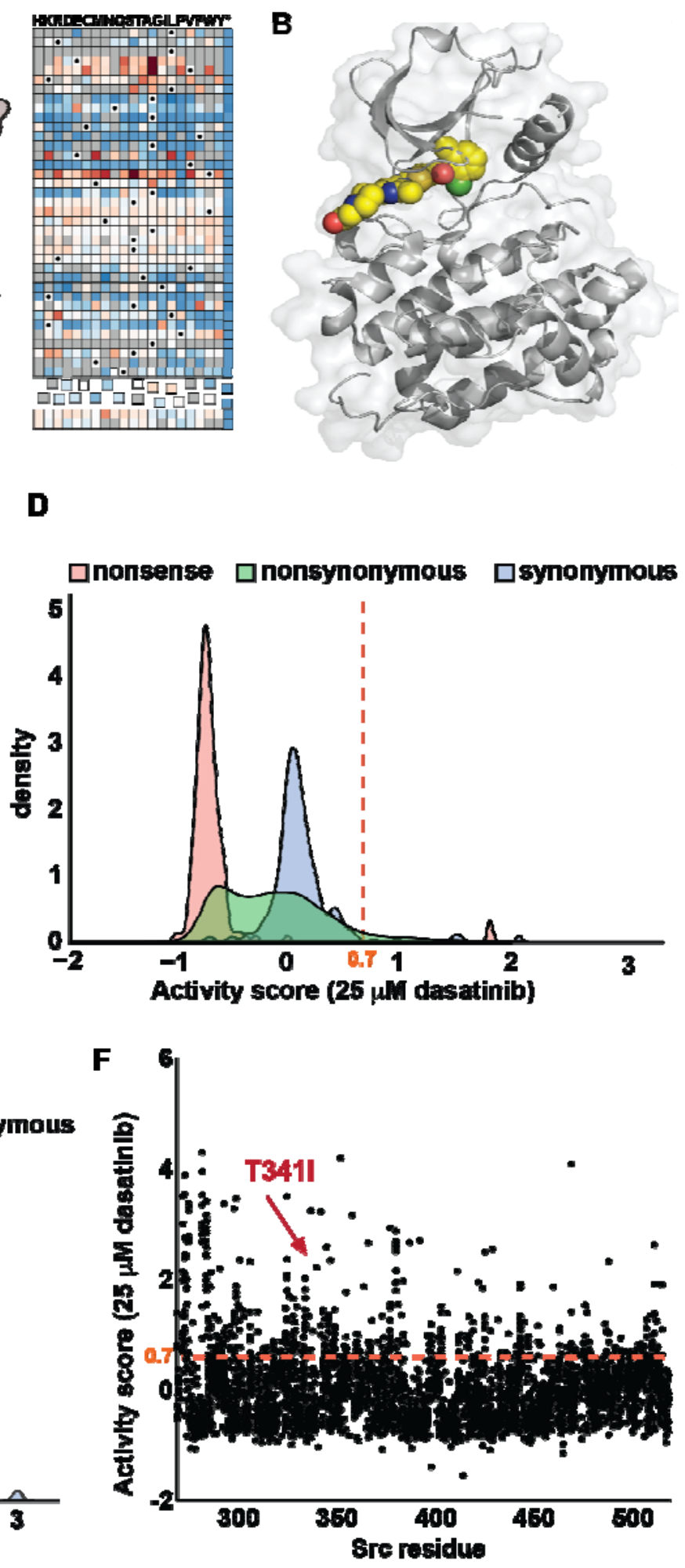

Figure 1. A yeast-based assay for the parallel analysis of drug resistance of Src tyrosine kinase mutants. (A) Schematic of the yeast-based growth assay used to quantify the drug resistance of thousands of Src mutants (B) Cocrystal structure of Src's catalytic domain (PDB ID: 3G5D) bound to dasatinib (colored spheres). (C) Scatterplot showing the correlation (Pearson's $R=0.85$ ) between the activity scores obtained in $25 \mu \mathrm{M}$ dasatinib-treated yeast for two independent transformations of the Src ${ }^{\text {myr }}$ variant library. (D, E) Activity score distributions of all synonymous and nonsynonymous (missense and nonsense) $\mathrm{Src}^{\text {myr }}$ variants in yeast treated with $25 \mu \mathrm{M}$ (D) or $100 \mu \mathrm{M}$ (E) 
dasatinib. The red dashed line indicates the activity score value (twice the standard deviation of the synonymous distribution) we defined as dasatinib resistant. (F) Scatterplot showing activity scores for every single amino acid substitution at every residue in Src's catalytic domain for yeast transformed with the Src ${ }^{\text {myr }}$ library and treated with 25 $\mu \mathrm{M}$ dasatinib. The red dashed line indicates the activity score value we defined as dasatinib resistant, and each black dot represents activity score derived from averaging two replicates.

Profiling of dasatinib resistance in Src. We assembled our large-scale mutagenesis data into a sequenceactivity map covering more than $75 \%$ of all possible single mutants of Src's catalytic domain (Figure 2 A, Table S3). About $12 \%$ of these single mutants were defined as dasatinib-resistant based on our classification criteria. Consistent with the notion that preservation of phosphotransferase activity is required for a mutation to confer drug resistance, we observed that almost all dasatinib-resistant mutations were either classified as activating $(\sim 65 \%)$ or WT-like $(\sim 30 \%)$ in a comprehensive analysis of Src's phosphotransferase activity that we previously performed (Figure 2B, S2A) (Ahler et al., 2019). While elevated phosphotransferase activity appears to be a common dasatinib resistance mechanism, activity scores obtained in the absence or presence of dasatinib were not strictly correlated (Figure 2B, S2B), suggesting that diverse mechanism of resistance were captured. Our sequence-activity map revealed that dasatinib-resistant mutations were distributed throughout the catalytic domain, especially in multiple residues in the $\beta 1$ and $\beta 2$ strands of the $N$-terminal lobe and the $\alpha \mathrm{D}$ and $\alpha E$ helices of the C-lobe (Figure 2A, 2B). Finally, at many positions only a few mutations conferred dasatinib resistance, while at a subset of residues most substitutions demonstrated dasatinib resistance (Figure 2A).

Mutations that perturb inhibitor contact residues in the ATP-binding pocket are a common mechanism of resistance to kinase inhibitors (Persky et al., 2020). We explored whether our large-scale mutagenesis data captured this mechanism of dasatinib resistance by mapping the maximum and mean activity scores that were obtained in the presence of $25 \mu \mathrm{M}$ dasatinib onto the fifteen residues in Src's ATP-binding site that interact with dasatinib (Figure 2C, 2D). As expected, mutations at a number of residues that line the ATP-binding pocket led to substantial dasatinib resistance, including the well-characterized T341I gatekeeper mutation (Azam et al., 2008; Krishnamurty and Maly, 2010). In addition to the gatekeeper residue, residues Leu276 (located on the $\beta 1$ strand), Val326 (located between the $\alpha \mathrm{C}$ helix and $\beta 4$ strand) and Tyr343 (located in the hinge region) all showed high position-averaged activity scores in the presence of $25 \mu \mathrm{M}$ dasatinib (Figure 2D, 2E) and appeared to be highly susceptible to the development of resistance. In total, at least one substitution led to resistance at two-thirds of the residues that interact with dasatinib, consistent with the ATP-binding pocket being a site of significant potential drug resistance. Our results are consistent with a recent screen of cyclindependent kinase 6 (CDK6)'s resistance to the ATP-competitive inhibitor palbociclib (Persky et al., 2020) (Figure S2D). Despite the slightly different binding poses of palbociclib and dasatinib within the ATP-binding pockets of CDK6 and Src, respectively, resistance mutations occurred at many of the same contact residues, and a similar percentage of contact residues were capable of acquiring inhibitor resistance (Figure S2C).

Our sequence-activity map shows that there are several residues where numerous substitutions led to dasatinib resistance, suggesting that they represent sites that are particularly prone to the development of drug resistance. To quantitatively identify resistance-prone sites, we determined all residues in Src's catalytic domain that demonstrated mean (position-averaged) activity scores greater than our defined drug-resistance cutoff for an individual mutant in the presence of $25 \mu \mathrm{M}$ dasatinib (Figure 2E, 2F, S2E). In total, twelve residues meet this definition of "resistance-prone" residues. While four of the twelve resistance-prone residues (Leu276, Val326, Thr341, Tyr343) interact with dasatinib (Figure 2C, 2D), the remaining eight either possess side-chains that are directed away from the ATP-binding site or are distal to the site of dasatinib binding. We previously observed that all eight of the resistance-prone residues that do not interact with dasatinib contained a large number of activating mutations in the absence of dasatinib and are likely involved in the regulation of Src's phosphotransferase activity (Ahler et al., 2019). Ala378 and Glu381 are components of the regulatory $\alpha \mathrm{F}$ pocket in the C-terminal lobe that contributes to the autoinhibition of Src's phosphotransferase activity through an interaction with the N-terminal SH4 domain. Resistance-prone residues Glu273, Val274, Lys275, Glu283, and Trp285 form a solvent-exposed, spatially-defined cluster of residues located on the $\beta 1$ and $\beta 2$ strands of the N-terminal lobe and appear to contribute to Src's autoinhibition by an unknown mechanism. Thus, elevated phosphotransferase activity appears to be a general mechanism for acquiring dasatinib resistance in Src. 


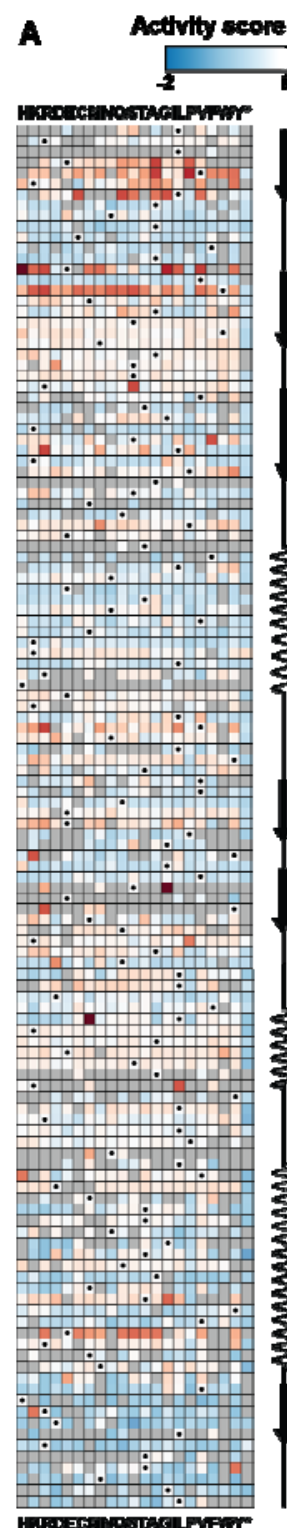

E

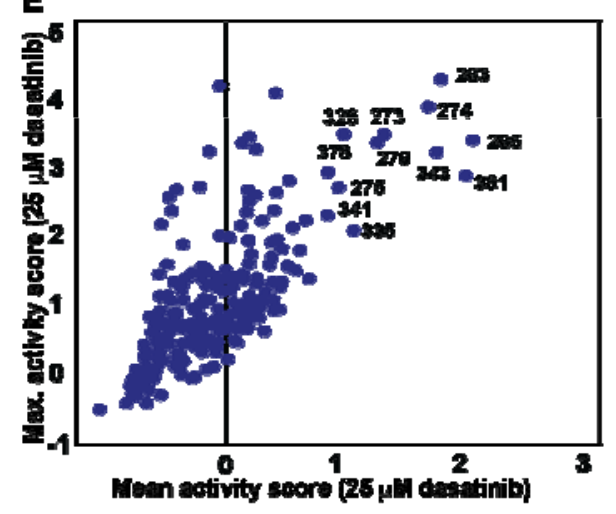

B
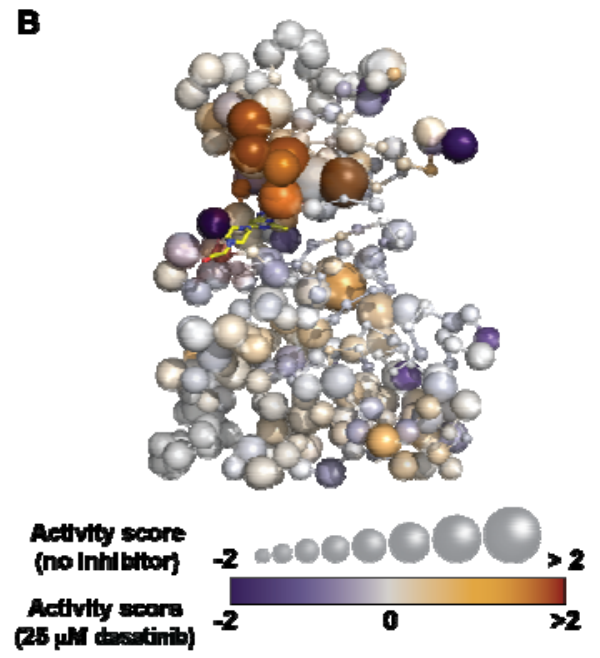

C
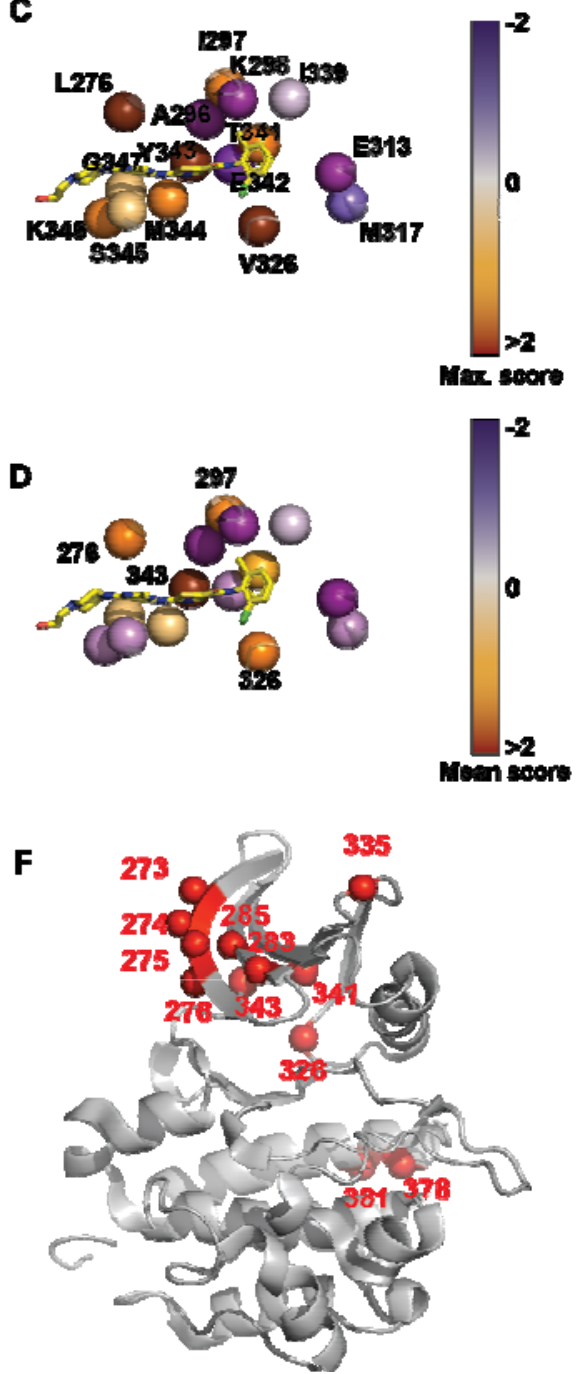

Figure 2. Mapping of dasatinib resistance mutations in Src. (A) Sequence-activity score map for all residues in Src's catalytic domain for the Src ${ }^{\text {myr }}$ variant library treated with $25 \mu \mathrm{M}$ dasatinib. Black dots represent the wild-type amino acid and gray tiles indicate missing data. Secondary structure and functional motif annotations were obtained from the ProKinO database. (B) Mean (position-averaged) activity scores for each residue in Src's catalytic domain (PDB ID: 3G5D) for the Src ${ }^{\text {myr }}$ variant library in yeast treated with DMSO. Sphere size indicates position-averaged 
activity scores in the absence of dasatinib (Ahler et al., 2019); color indicates position-averaged activity scores in the presence of $25 \mu \mathrm{M}$ dasatinib (this work). Nonsense mutants were excluded from the average score. (C) Co-crystal structure (PDB ID: 3G5D) of Src's catalytic domain bound to dasatinib (yellow sticks) showing maximum activity scores in the presence of $25 \mu \mathrm{M}$ dasatinib for all residues that interact with dasatinib. (D) Co-crystal structure (PDB ID: 3G5D) of Src's catalytic domain bound to dasatinib (yellow sticks) showing position-averaged activity scores in the presence of $25 \mu \mathrm{M}$ dasatinib for all residues that interact with dasatinib. (E) Correlation plot of maximum activity scores and mean activity scores in the presence of $25 \mu \mathrm{M}$ dasatinib for each residue in Src's catalytic domain. ( $F$ ) Crystal structure (PDB ID: 3G5D) of Src's catalytic domain with the twelve residues that were defined as "resistance prone" shown as red spheres.

Src mutational resistance to different modes of ATP-competitive inhibition. We further explored the intersection between the regulation of Src's phosphotransferase activity and drug resistance by determining how resistance arises in response to different modes of ATP-competitive inhibition (Figure S3A) (Ranjitkar et al., 2010)(Fang 2020). We profiled our Src variant library in the presence of each of a matched set of pyrazolopyrimidine-based ATP-competitive inhibitors that stabilize structurally distinct conformations of Src's ATP-binding site (Figure 3A). Inhibitor 1 contains a 3-phenol at the C-3 position of the pyrazolopyrimidine scaffold that can potentially form a hydrogen-bond with the side-chain of Glu313 in the helix $\alpha \mathrm{C}$, leading to stabilization of the active $\alpha \mathrm{C}$ helix-in conformation (Figure S3A). Inhibitor 2 contains a pharmacophore at the C-3 position that promotes the outward rotation of helix $\alpha \mathrm{C}$ to an inactive conformation (helix $\alpha \mathrm{C}$-out). We also attempted to profile an inhibitor (inhibitor 3) that contains a pharmacophore at the C-3 position of the pyrazolopyrimidine scaffold that stabilizes a flipped, inactive conformation of Src's activation loop (DFG-out) but is otherwise identical to inhibitors $\mathbf{1}$ and $\mathbf{2}$, but it did not achieve sufficiently high intracellular concentrations in yeast to inhibit WT Src ${ }^{\text {myr }}$. Therefore, we used inhibitor 4, an analog of 3 that achieved high enough concentrations in yeast to inhibit WT Src ${ }^{\text {myr }}$ (Figure 3A), for our profiling of conformation-selective inhibitor resistance.

After transforming the Src ${ }^{\text {myr }}$ variant library into yeast, we performed outgrowth in the presence of either inhibitor 1, 2, or $\mathbf{4}$ and collected samples at various time points. For each inhibitor, we calculated normalized activity scores for $\sim 3,500$ Src mutants (Figure 3B, Table S4). As for dasatinib, a Src variant was defined as being resistant if its activity score in the presence of a specific conformation-selective inhibitor was greater than two times the standard deviation of the synonymous distribution. Using this definition, we identified 107, 86, and 114 Src mutants that were resistant to inhibitors 1, 2, and 4, respectively (Figure S3B). Similar to Src's resistance to dasatinib, almost all variants that were resistant to inhibitors $\mathbf{1 , 2}$, or $\mathbf{4}$ were either classified as activating or WT-like in our previous analysis of Src's phosphotransferase activity (Figure S3C). In comparing the overlap between resistance mutations, we observed 22 mutations occurring at eight residues that were resistant to all three conformation-selective inhibitors (Figure 3C, S3D). Furthermore, inhibitors $\mathbf{1}$ and $\mathbf{4}$ shared the highest overlap ( 75\%) in resistance mutations, suggesting that their shared ability to stabilize an active conformation of Src's $\alpha \mathrm{C}$ helix makes them susceptible to similar mechanisms of resistance. Despite these similarities, we observed that $\sim 25-45 \%$ of all resistance mutations for a particular inhibitor were unique (Figure 3D), revealing resistance mechanisms that are likely specific to different modes of conformation-selective inhibition.

To provide a comprehensive overview of which regions are generally resistant to different modes of ATPcompetitive inhibition, we mapped position-averaged activity scores for each conformation-selective inhibitor onto the catalytic domain of Src (Figure 3E). Inhibitors $\mathbf{1}$ and $\mathbf{4}$ shared similar patterns of resistance, and the solvent-facing $\mathrm{N}$-terminal lobe of the catalytic domain was the main region of resistance. While residues in the same region of Src's N-terminal lobe also demonstrated resistance to inhibitor $\mathbf{2}$, unique resistance mutations to this helix $\alpha \mathrm{C}$-out-stabilizing inhibitor were also observed through the entirety of Src's kinase domain. In particular, three residues (Leu300, lle337, and Leu413) contained multiple mutations that uniquely conferred strong resistance to inhibitor 2. Leu300 is located on the linker that connects the $\alpha \mathrm{C}$ helix to the $\beta$-sheet of the $\mathrm{N}$-terminal lobe and lle337 is located on the face of the $\beta 4$ strand that is directed towards helix $\alpha \mathrm{C}$. A plausible explanation for how mutations at these residues confer resistance is that they negatively influence the ability of helix $\alpha C$ to adopt the inactive "out" conformation that is required for inhibitor $\mathbf{2}$ to be accommodated in Src's ATP-binding pocket.

We observed six residues (Leu276, Trp285, Val326, Thr341, Tyr343, and Glu381) that fulfill our definition of being resistance-prone to all three conformation-selective inhibitors (Figure 3E, S3D). These six residues were also classified as being resistance-prone for dasatinib (Figure 2F). The side-chains of Leu276, Val326, Thr341, 
bioRxiv preprint doi: https://doi.org/10.1101/2021.12.05.471322; this version posted January 3, 2022. The copyright holder for this preprint (which was not certified by peer review) is the author/funder. All rights reserved. No reuse allowed without permission.

and Tyr343 are all directed towards Src's ATP-binding pocket and mutations at these residues most likely confer resistance by perturbing inhibitor contacts. For example, Thr341 is commonly referred to as the gatekeeper residue and many substitutions at this residue have been shown to lead to inhibitor resistance by restricting the size of the ATP-binding pocket. We speculated that mutations at Val326 (Figure S3E), which contains a side-chain that is directed towards the adenine ring of ATP, provided resistance through a similar mechanism. To test this, we performed phosphotransferase activity and inhibition assays with WT and an inhibitor-resistant variant (V326K) of purified full-length $\mathrm{Src}\left(\mathrm{Src}^{\mathrm{FL}}\right)$. We observed that purified $\mathrm{V} 326 \mathrm{~K} \mathrm{Src}{ }^{\mathrm{FL}}$ 's $\mathrm{K}_{\mathrm{M}}$ for ATP ( $\left.\mathrm{K}_{\mathrm{M}}[\mathrm{ATP}]\right)$ and phosphotransferase activity were very similar to WT Src ${ }^{\mathrm{FL}} \mathrm{s}$ (Figure S3F). Consistent with the V326K mutation leading to reduced inhibitor affinity, purified V326K $\mathrm{Src}^{\mathrm{FL}}$ displayed $\mathrm{K}_{\mathrm{I}}$ values for inhibitors 1, 2 and 3 that were 5- to 50-fold higher than WT Src ${ }^{\mathrm{FL}}$ (Figure 3F). Interestingly, the tyrosine kinases BCR-Abl, Alk, and c-KIT also contain a valine at an equivalent position in their ATP-binding sites and drugresistant mutations have been observed at this position in the clinic (Table S3). Thus, this region of the ATPbinding site appears to be a site of inhibitor resistance for a number of tyrosine kinases. 
A

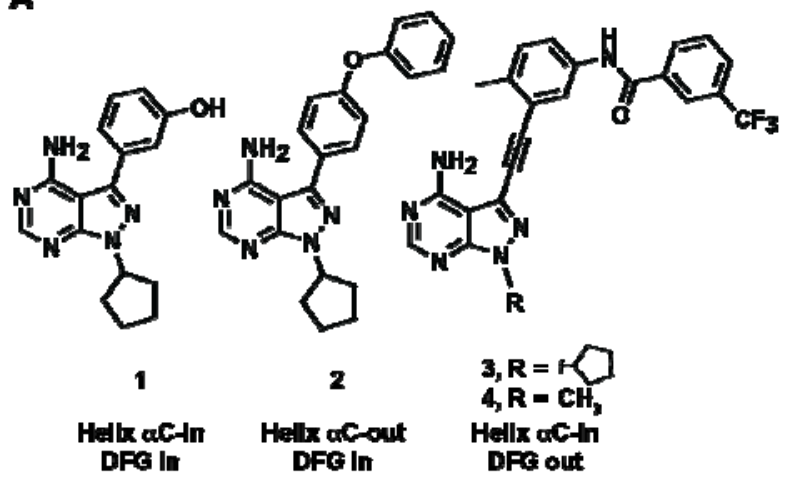

B

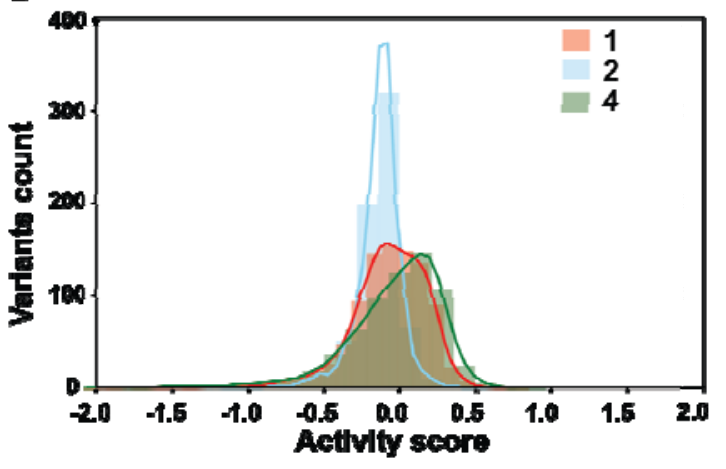

c

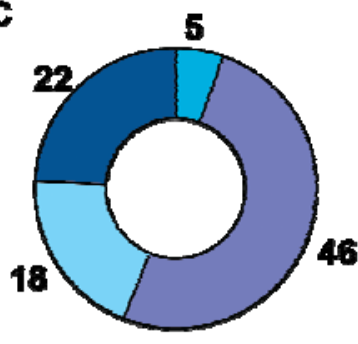

$\square 122 \quad \square 284$
184 $\square$ 1,2,84
Shared varlants

D

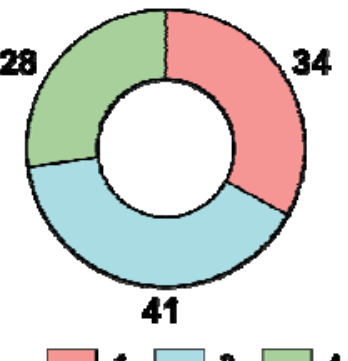

Unlque varlants

E
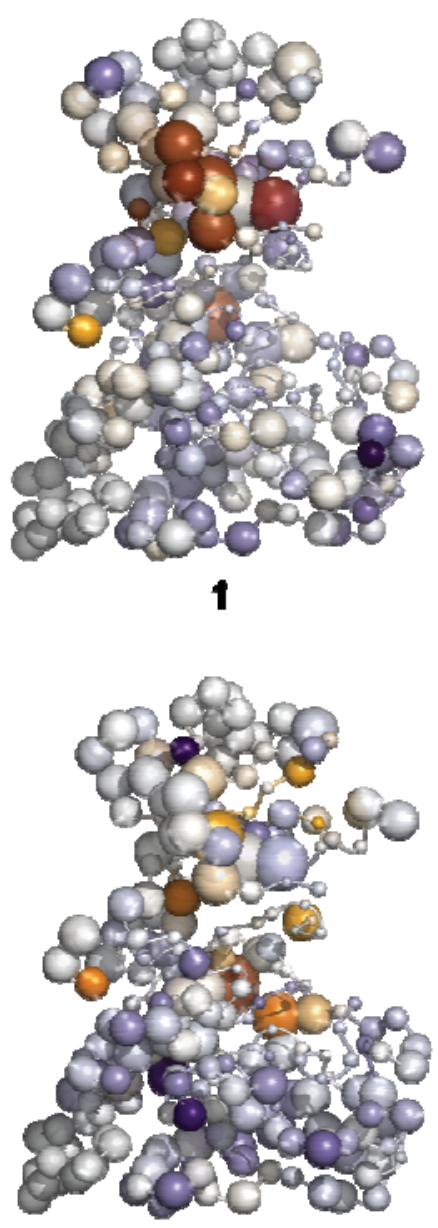

2

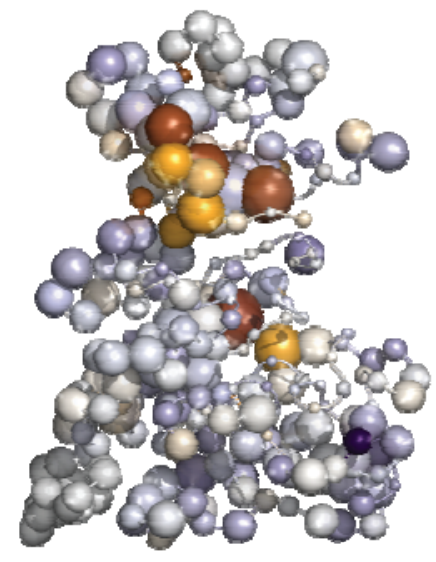

4

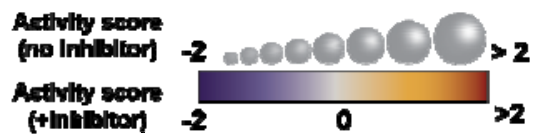


bioRxiv preprint doi: https://doi.org/10.1101/2021.12.05.471322; this version posted January 3, 2022. The copyright holder for this preprint (which was not certified by peer review) is the author/funder. All rights reserved. No reuse allowed without permission.

Figure 3. Parallel analysis of mutations conferring Src resistance to conformation-selective, ATP-competitive inhibitors. (A) Structures of the conformation-selective, ATP-competitive inhibitors that were profiled in our yeastbased growth assay. (B) Activity score distribution of all non-synonymous Src variants in yeast treated with inhibitors 1, 2 or 4. (C) Donut plot showing the number of shared inhibitor-resistant mutants. (D) Donut plot showing the number of unique inhibitor-resistant mutants for inhibitors 1, 2 and 4. (E) Position-averaged activity scores for each residue in Src's catalytic domain (PDB ID: 3G5D) for the Src ${ }^{\text {myr }}$ variant library treated with conformation-selective inhibitor 1 (top), 2 (middle) or 4 (bottom) and in absence of inhibitor (!!! INVALID CITATION !!! (data from Ahler et al., 2019)). Position-averaged activity scores in the presence of each inhibitor are depicted by color, while activity scores in the absence of inhibitors are represented by sphere size. $(\mathrm{F}) K_{l}$ values $(\mathrm{n}=3$, mean $\pm \mathrm{SEM}$ ) of conformation-selective inhibitors 1-3 for WT and V326K Src ${ }^{\mathrm{FL}}$.

Characterization of a drug-resistant cluster of mutants in the N-terminal lobe of Src's catalytic domain. The side-chains of six residues (Glu273, Val274, Lys275, Leu276, Glu283, and Trp285) that possess multiple drug-resistant mutants form a spatially-defined cluster on the top face of the $\beta 1$ and $\beta 2$ stands in the $\mathrm{N}$-terminal lobe of Src's catalytic domain (Figure 4A, S4A). Despite the high number of mutations that conferred resistance at the six residues within this cluster, which we hereafter refer to as the $\beta 1 / \beta 2$ resistance cluster, the side-chains of all but Leu276 are solvent exposed and directed away from the site of ATP binding in Src (Figure S4B). Therefore, we were curious why this cluster of solvent-exposed residues is so prone to the development of drug resistance. To determine whether mutations within the $\beta 1 / \beta 2$ resistance cluster confer resistance by reducing Src's affinity for ATP-competitive inhibitors, we determined the $K_{1}$ values of inhibitors 1-3 for purified $\mathrm{Src}^{\mathrm{FL}}$ constructs containing drug-resistant E283M or W285T mutations (Figure 4B). We found that the presence of either the E283M or W285T mutation led to increased $K_{1}$ values for inhibitors 1-3 relative to WT $\mathrm{Src}^{\mathrm{FL}}$, with the highest level of resistance observed for DFG-out-stabilizing inhibitor 3 . Furthermore, we found that both mutations conferred similar levels of resistance. Therefore, mutations in the $\beta 1 / \beta 2$ resistance cluster confer resistance, in part, by lowering the affinity of inhibitors for Src's ATP-binding site. 
A

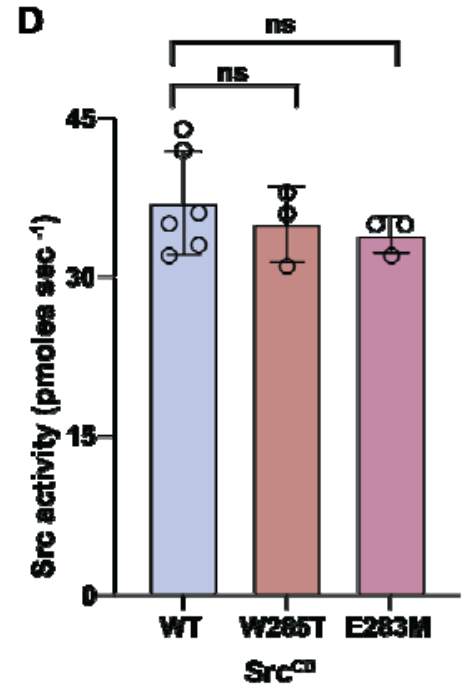

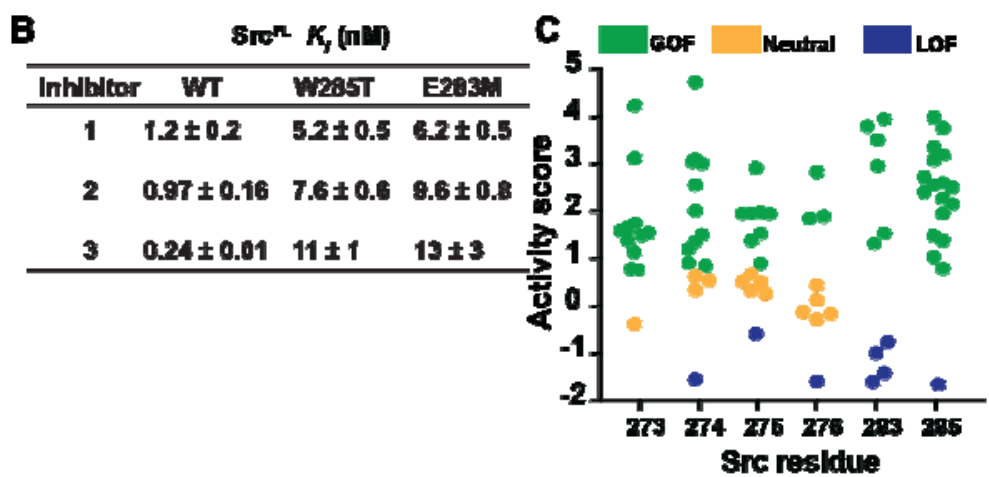

$\mathbf{E}$
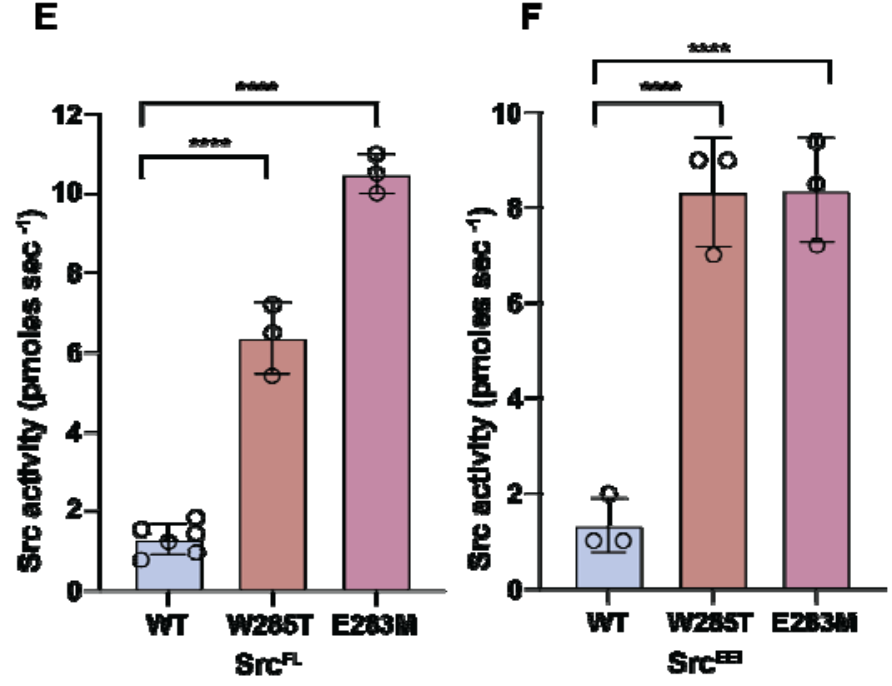

G

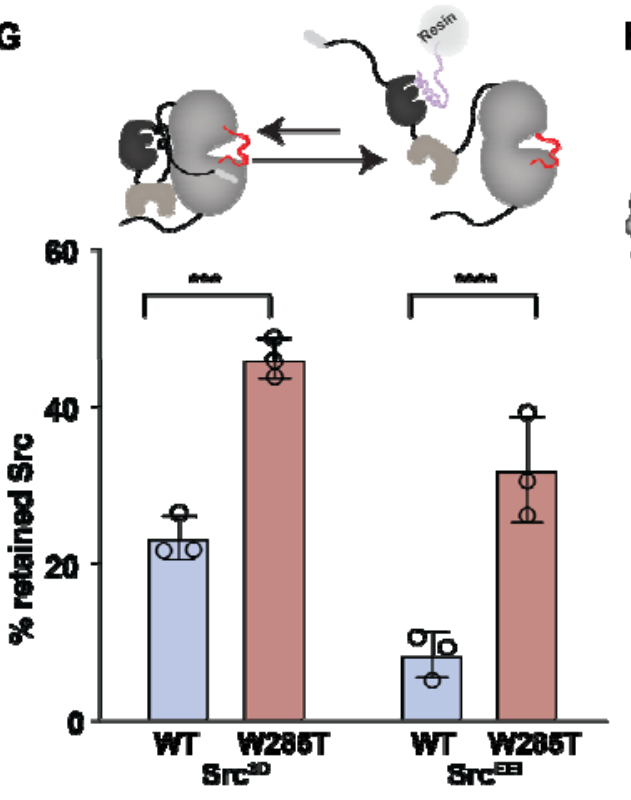

H

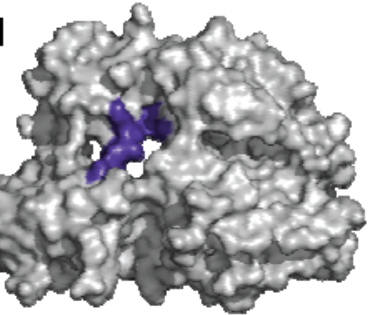

'closed'

less sensittive to llmlied proteolysis

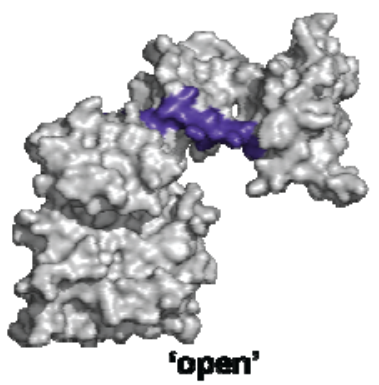

more sansitiva to limitod proteolysis
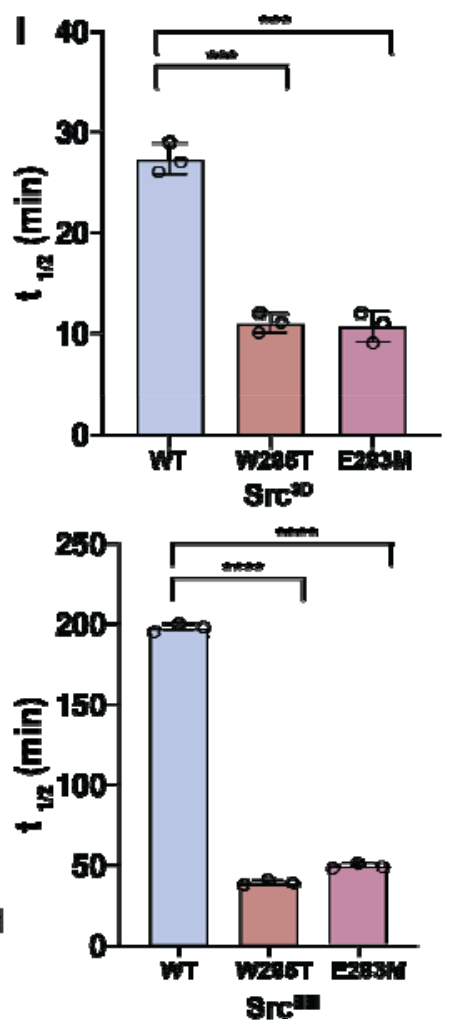
Figure 4. Biochemical characterization of the $\beta 1 / 2$ resistance cluster. (A) Top-down view of the $N$-terminal lobe of Src's catalytic domain (PDB ID: 1Y57) with the side-chains of all six residues in the $\beta 1 / 2$ resistance cluster shown in orange. (B) $K_{l}$ values of inhibitors 1-3 for the WT, E283M and W285T mutants of Src ${ }^{\mathrm{FL}}(\mathrm{n}=3$, mean $\pm \mathrm{SEM}$,). (C) Activity scores for every substitution at each residue in the $\beta 1 / 2$ resistance cluster in the absence of an inhibitor. Values were determined in a previous study (Ahler et al., 2019). (D) Phosphotransferase activity of purified WT, W285T, or E283M Src ${ }^{C D}(n=3-5, n s=$ non-significant, $P>0.05)$. (E) Phosphotransferase activity of purified WT, W285T, or E283M Src ${ }^{\mathrm{FL}}\left(\mathrm{n}=3-5,{ }^{\star \star \star *} P<0.0001\right)$. (F) Phosphotransferase activity of purified WT, W285T or E283M $\operatorname{Src}^{\mathrm{EEI}}\left(\mathrm{n}=3,{ }^{* * *} P<0.0001\right)$. (G) Schematic of the $\mathrm{SH} 3$ domain pull-down assay (top). To detect global conformation, Src constructs were incubated with an immobilized SH3 domain ligand. Closed, SH3-engaged Src is unable to bind to the resin, whereas open, SH3-disengaged Src binds. After washing, retained Src is eluted and quantified by western blot analysis (bottom). Percent retained WT or W285T Src ${ }^{3 \mathrm{D}}$ (left, $n=3,{ }^{* * *} P<0.001$ ) and WT or W285T Src ${ }^{\mathrm{EEI}}$ (right, $\mathrm{n}=3,{ }^{* \star \star *} P<0.0001$ ) in the SH3 domain pull-down assay. (H) Crystal structure (PDB ID: 2SRC) of Src ${ }^{3 \mathrm{D}}$ in the closed (top) and open (bottom) global conformations. The SH2-catalytic domain linker (violet) of the open conformation of $\mathrm{Src}^{3 \mathrm{D}}$ is more sensitive to proteolysis by thermolysin than the closed global conformation. (I) Half-life values of purified WT, E283M, or W285T Src ${ }^{3 \mathrm{D}}$ (top, $\left.\mathrm{n}=3,{ }^{* * *} P<0.001\right)$ and WT, E283M, or W285T Src ${ }^{\mathrm{EEI}}$ (bottom, $\mathrm{n}=3,{ }^{* * * *} P<$ $0.0001)$ in the thermolysin limited proteolysis assay.

Residues in the $\beta 1 / \beta 2$ resistance cluster modulate autoinhibition of Src. We previously observed that all six residues within the $\beta 1 / \beta 2$ resistance cluster also contained multiple activating mutations in the absence of inhibitors (Figure 4C), suggesting that this region may serve a role in modulating Src's phosphotransferase activity (Ahler et al., 2019). Therefore, we next determined how activating mutations in the $\beta 1 / \beta 2$ resistance cluster influence Src's phosphotransferase activity with purified Src constructs (Figure S4C). Interestingly, we found that the E283M and W285T mutations had a negligible effect on the phosphotransferase activity of a construct $\left(\mathrm{Src}^{\mathrm{CD}}\right)$ consisting solely of the catalytic domain of Src (Figure 4D, S4D). However, we observed that both mutations were activating in the context of $\mathrm{Src}^{\mathrm{FL}}$ (Figure 4E), suggesting that activating mutations in the $\beta 1 / \beta 2$ resistance cluster release regulatory domain-mediated autoinhibition. Consistent with the $\beta 1 / \beta 2$ resistance cluster influencing regulation mediated by Src's SH2 and SH3 domains, and not the N-terminal SH4 and unique domains, the E283M and W285T mutations showed a similar level of activation relative to WT in a $\mathrm{Src}^{3 \mathrm{D}}$ construct, Src ${ }^{\mathrm{EE}}$, that possesses $\mathrm{SH} 2$ domain interaction-enhancing mutations in the C-terminal tail that promote a similar level of autoinhibition as $\mathrm{Src}^{\mathrm{FL}}$ (Figure 4F). Together, our data support a model wherein residues in the $\beta 1 / \beta 2$ resistance cluster reinforce the autoinhibition provided by the $\mathrm{SH} 2$ and $\mathrm{SH} 3$ domain regulatory apparatus, which activating mutations release.

We speculated that activating mutations in the $\beta 1 / \beta 2$ resistance cluster increase Src's phosphotransferase activity by reducing levels of intramolecular $\mathrm{SH} 2$ and $\mathrm{SH} 3$ domain regulatory engagement. To test this prediction, we assessed how mutations affect intramolecular regulatory domain engagement levels with two biochemical assays. First, we measured intramolecular $\mathrm{SH} 3$ domain engagement levels with an immobilized $\mathrm{SH} 3$ domain ligand pull-down assay (Figure 4G). Consistent with activating $\beta 1 / \beta 2$ resistance cluster mutations leading to increased phosphotransferase activity by reducing the autoinhibitory engagement of Src's regulatory apparatus, W285T $\mathrm{Src}^{3 \mathrm{D}}$ 's association with an immobilized SH3 domain ligand was more than 2-fold greater than WT Src ${ }^{3 \mathrm{D}}$ 's (Figure 4G, S4E). Furthermore, the W285T mutation dramatically increased the ability of the more regulatory domain-engaged $\mathrm{Src}^{\mathrm{EEI}}$ construct to intermolecularly interact with the immobilized $\mathrm{SH} 3$ domain ligand relative to WT Src ${ }^{\mathrm{EET}}$ (Figure 4G, S4E). We next used the rate of proteolysis of the flexible linker that connects Src's SH2 domain to its CD (SH2-CD linker) by the metalloprotease thermolysin to characterize how $\beta 1 / \beta 2$ resistance cluster mutations affect intramolecular regulatory domain engagement. Previous studies have demonstrated an inverse correlation between the rate of thermolysin cleavage, measured as intact protein halflife $\left(t_{1 / 2}\right)$, of the $\mathrm{SH} 2-\mathrm{CD}$ linker and intramolecular $\mathrm{SH} 2$ and $\mathrm{SH} 3$ regulatory domain engagement levels (Figure 4H) (Agius et al., 2019; Fang et al., 2020). Concordant with the SH3 domain pull-down results, the SH2-CD linkers of E283M and W285T Src ${ }^{3 \mathrm{D}}$ were proteolyzed $\sim 3$ times more rapidly than WT Src ${ }^{3 \mathrm{D}}$ (Figure 4I, S4F). The E283M and W285T mutations also dramatically increased the rate of proteolytic cleavage of Src ${ }^{\mathrm{EEl}}$ 's SH2CD linker (Figure $\mathbf{4 I}, \mathbf{S 4 G}$ ). Thus, residues in the $\beta 1 / \beta 2$ resistance cluster appear to influence the level of intramolecular engagement of Src's SH2 and SH3 domains. 

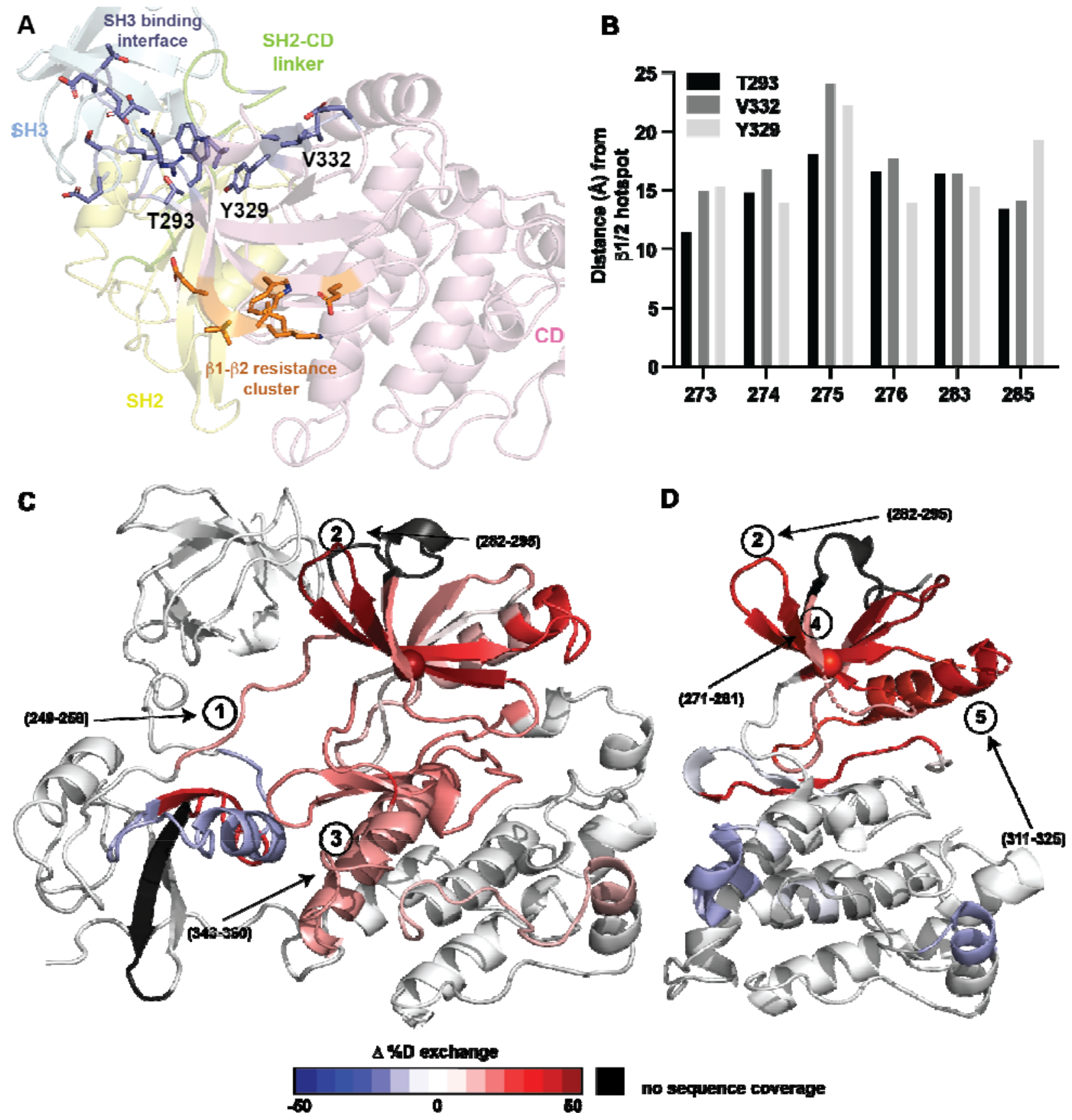

Figure 5. Conformational dynamics of W285T Src. (A) Structure of autoinhibited Src ${ }^{3 D}$ (PDB ID: 2SRC) showing the side-chains of residues in the $\beta 1 / \beta 2$ resistance cluster (orange) and the side-chains of residues in the nearest regulatory interface (blue, SH3 domain interface) in Src's catalytic domain. (B) Plot showing the distances between the $\beta$-carbons of the three residues (T293, Y329, and V332) in the SH3 domain regulatory interface closest to the $\beta 1 / 2$ resistant cluster and the $\beta$-carbons of residues in the $\beta 1 / 2$ resistant cluster. (C) HDX-MS analysis of purified WT and W285T Src ${ }^{3 \mathrm{D}}$. Deuteration differences between WT and W285T Src ${ }^{3 \mathrm{D}}$ are plotted on the crystal structure of Src ${ }^{3 \mathrm{D}}$ (PDB ID: 2SRC). Regions that show increased deuterium uptake in W285T Src ${ }^{3 \mathrm{D}}$ relative to WT Src ${ }^{3 \mathrm{D}}$ are shown in red, while regions that show no difference are shown in white. $(n=3$, mean \pm SEM) (D) HDX-MS analysis of purified WT and W285T Src ${ }^{C D}$. Deuteration differences between WT and W285T Src ${ }^{C D}$ are plotted on the crystal structure of Src's catalytic domain (PDB ID: 3G5D). Regions that show increased deuterium uptake in W285T Src ${ }^{\mathrm{CD}}$ relative to WT $\mathrm{Src}^{\mathrm{CD}}$ are shown in red, while regions that show no difference are shown in white. $(\mathrm{n}=3$, mean $\pm \mathrm{SEM})$. 
The W285T mutation promotes a more dynamic N-terminal lobe of Src. While our biochemical analyses suggest that activating mutations in the $\beta 1 / \beta 2$ resistance cluster increase Src's phosphotransferase activity by promoting a more open, regulatory domain-disengaged global conformation, the mechanistic basis for this effect was unclear. To the best of our knowledge, no previous structural or biochemical studies have suggested that residues within the $\beta 1 / \beta 2$ resistance cluster participate in regulatory interactions. Furthermore, residues in the $\beta 1 / \beta 2$ resistance cluster are separated by $>10 \AA$ from the nearest regulatory interface of autoinhibited Src (Figure 5A, 5B). Thus, we performed Hydrogen-Deuterium eXchange Mass Spectrometry (HDX-MS) on Src ${ }^{3 \mathrm{D}}$ to provide an unbiased analysis of how activating $\beta 1 / \beta 2$ resistance cluster mutations affect the global conformational dynamics of Src. Specifically, we compared the deuterium backbone exchange kinetics of W285T $\mathrm{Src}^{3 \mathrm{D}}$ relative to WT Src ${ }^{3 \mathrm{D}}$. We subjected identical concentrations of WT $\mathrm{Src}^{3 \mathrm{D}}$ and W285T $\mathrm{Src}^{3 \mathrm{D}}$ to standard $\mathrm{D}_{2} \mathrm{O}$ exchange conditions and samples were quenched and processed at various time points using established methods. Using this protocol, we were able to monitor the exchange kinetics of peptic peptides covering $\sim 85 \%$ of WT Src ${ }^{3 \mathrm{D}}$ 's and W285T Src ${ }^{3 \mathrm{D}}$ 's sequence (Table S5).

Mapping differences in deuterium exchange kinetics between W285T $\mathrm{Src}^{3 \mathrm{D}}$ and WT $\mathrm{Src}^{3 \mathrm{D}}$ onto a structure of autoinhibited $\mathrm{Src}^{3 \mathrm{D}}$ (Figure 5C) revealed that $\sim 50 \%$ of residues in W285T $\mathrm{Src}^{3 \mathrm{D}}$ underwent faster exchange kinetics than in WT Src ${ }^{3 D}$. Consistent with $\beta 1 / \beta 2$ resistance cluster mutations leading to a reduction in the intramolecular engagement of Src's regulatory SH2 and SH3 domain apparatus (Figures 4G, 4I), peptic peptides covering the $\mathrm{SH} 2$ and $\mathrm{SH} 3$ domain interfaces of autoinhibited Src (peptides 1 and 3, Figure 5C) demonstrated increased solvent accessibility in W285T Src ${ }^{3 \mathrm{D}}$ relative to WT Src ${ }^{3 \mathrm{D}}$. Furthermore, W285T also increased exchange kinetics in the activation loop and most of the N-terminal lobe of Src's catalytic domain. Thus, $\beta 1 / \beta 2$ resistance cluster mutations appear to lead to an increase in phosphotransferase activity by promoting a more open, regulatory domain-disengaged global state of Src and a dramatically more dynamic $\mathrm{N}$ terminal lobe of Src's catalytic domain.

The large number of peptic peptides that displayed faster exchange kinetics in W285T Src ${ }^{3 \mathrm{D}}$ relative to WT $\mathrm{Src}^{3 \mathrm{D}}$ made it difficult to discriminate which differences resulted from localized effects on dynamics versus those that arose from a more open and regulatory domain-disengaged conformational state of Src ${ }^{3 \mathrm{D}}$. Therefore, we performed a comparative HDX-MS analysis with Src constructs (W285T Src ${ }^{\mathrm{CD}}$ and WT Src ${ }^{\mathrm{CD}}$ ) that lack the $\mathrm{SH} 2$ and $\mathrm{SH} 3$ domain regulatory apparatus. We observed that the W285T mutation led to faster exchange kinetics for a smaller region of Src's catalytic domain in $\mathrm{Src}^{\mathrm{CD}}$ than in the context of $\mathrm{Src}^{3 \mathrm{D}}$, with most differences between W285T Src ${ }^{\mathrm{CD}}$ and WT Src ${ }^{\mathrm{CD}}$ confined to the N-terminal lobe (Figure 5D, S5). Similar to Src ${ }^{3 \mathrm{D}}$, the peptic peptide (peptide 2) that contains the W285T mutation (peptide 2, Figure 5C, 5D, S5) demonstrated markedly increased exchange kinetics in W285T Src ${ }^{\mathrm{CD}}$ relative to WT Src ${ }^{\mathrm{CD}}$. In addition to peptic peptide 2, we found that the peptic peptides that comprise the remainder of the catalytic domain's N-terminal lobe (Figure 5D, S5) also demonstrated greatly increased exchange kinetics in W285T Src ${ }^{\mathrm{CD}}$, suggesting that this mutation directly increases the dynamics of this entire region. Interestingly, a peptic peptide spanning a large portion of Src's helix $\alpha C$ demonstrated the largest difference in dynamics between W285T Src ${ }^{\mathrm{CD}}$ and WT Src ${ }^{\mathrm{CD}}$ (peptide 5 , Figure 5D, S5). Given the requirement that Src's helix $\alpha \mathrm{C}$ must adopt an ordered 'out' conformation for the catalytic domain to form a high affinity interaction with the $\mathrm{SH} 2$-catalytic domain linker and the $\mathrm{SH} 3$ domain in the autoinhibited form of Src, the ability of activating mutations in the $\beta 1 / \beta 2$ resistance cluster to directly increase the dynamics of this region provides a plausible mechanism for the reduced intramolecular regulatory domain engagement we observed in our biochemical assays (Figure 4). Together, our results support a model where residues in the $\beta 1 / \beta 2$ resistance cluster promote autoinhibitory interactions by restricting the conformational flexibility of the $\mathrm{N}$-terminal lobe, including the helix $\alpha \mathrm{C}$, which activating substitutions release, resulting in reduced autoinhibition and a subsequent increase in phosphotransferase activity.

Mutations in the $\beta 1 / 2$ resistance cluster affect the dynamics of Src's P-loop. Residues in the $\beta 1 / \beta 2$ resistance cluster are part of a structured region that flanks Src's phosphate-binding loop (P-loop, residues 277-282); a flexible catalytic element that coordinates the $\gamma$-phosphate of ATP (Figure 6A). A previous study showed that a salt bridge between $\beta 1 / \beta 2$ resistance cluster residues K252 and E260 in the Src Family Kinase (SFK) Lyn (equivalent to K275 and E283 in Src, respectively) appears to affect the dynamics of its P-loop and that mutations that disrupt this electrostatic interaction modulate Lyn's phosphotransferase activity (BarouchBentov et al., 2009). All SFKs contain amino acids that can potentially form a salt bridge at positions equivalent to K275 and E283 in Src (Figure 6B) and we observed that, like Src ${ }^{\text {myr }}$, a salt bridge-disrupting mutation (E260M) increased the activity of Lyn ${ }^{\text {myr }}$ relative to its WT variant in yeast (Figure 6C, S6A). Consistent with the potential importance of the salt bridge between K275 and E283 in influencing Src's phosphotransferase 
bioRxiv preprint doi: https://doi.org/10.1101/2021.12.05.471322; this version posted January 3, 2022. The copyright holder for this preprint (which was not certified by peer review) is the author/funder. All rights reserved. No reuse allowed without permission.

activity, we found that Src constructs that contain a mutation (E283D) that preserves, or potentially enhances, an electrostatic interaction with K275 showed reduced catalytic activity in yeast (Figure 6C, S6A) and in in vitro activity assays with purified Src constructs relative to WT (Figure 6D). This reduction in phosphotransferase activity of E283D Src ${ }^{3 \mathrm{D}}$ correlated with increased levels of regulatory domain engagement (Figure 6D, S6B). Together, previous data and our results suggest that a conserved salt bridge located within the $\beta 1 / \beta 2$ resistance cluster affects the dynamics of SFK P-loops, which influences their phosphotransferase activities.

We speculated that activating mutations at other positions in the $\beta 1 / \beta 2$ resistance cluster could also influence the dynamics of Src's P-loop like salt bridge-disrupting mutations. Indeed, our HDX-MS results demonstrated that the two peptic peptides that span the entirely of Src's P-loop showed greatly increased exchange dynamics in W285T Src ${ }^{3 \mathrm{D}}$ and $\mathrm{Src}^{\mathrm{CD}}$ (Figure 5C, 5D). However, the large size of the peptic peptides obtained in our HDX-MS workflow made it difficult to determine whether the increase in dynamics we observed was in the Ploop and/or in the structured $\beta$-sheet comprising Src's N-terminal lobe. Therefore, we used a chemical labeling methodology to probe the dynamics of a cysteine residue (Cys280) located in the center of Src's P-loop. Specifically, we compared the rates of N-ethylmaleimide (NEM) labeling of Cys280 in WT Src ${ }^{\mathrm{CD}}$ and W285T $\mathrm{Src}^{\mathrm{CD}}$. Consistent with the notion that activating mutations in the $\beta 1 / \beta 2$ resistance cluster modulate the dynamics of Src's P-loop, Cys280 in W285T Src ${ }^{C D}$ showed a significantly increased rate of NEM labeling relative to WT Src ${ }^{\mathrm{CD}}$ (Figure 6E). Thus, activating mutations in the $\beta 1 / \beta 2$ resistance cluster not only increase the overall dynamics of the catalytic domain's N-terminal lobe but also increase the dynamics of the P-loop. We speculate that residues in the $\beta 1 / \beta 2$ resistance cluster act as a network that couples the dynamics of the P-loop to autoinhibitory SH2/SH3 interactions that occur on the opposite face of Src's catalytic domain. 
A
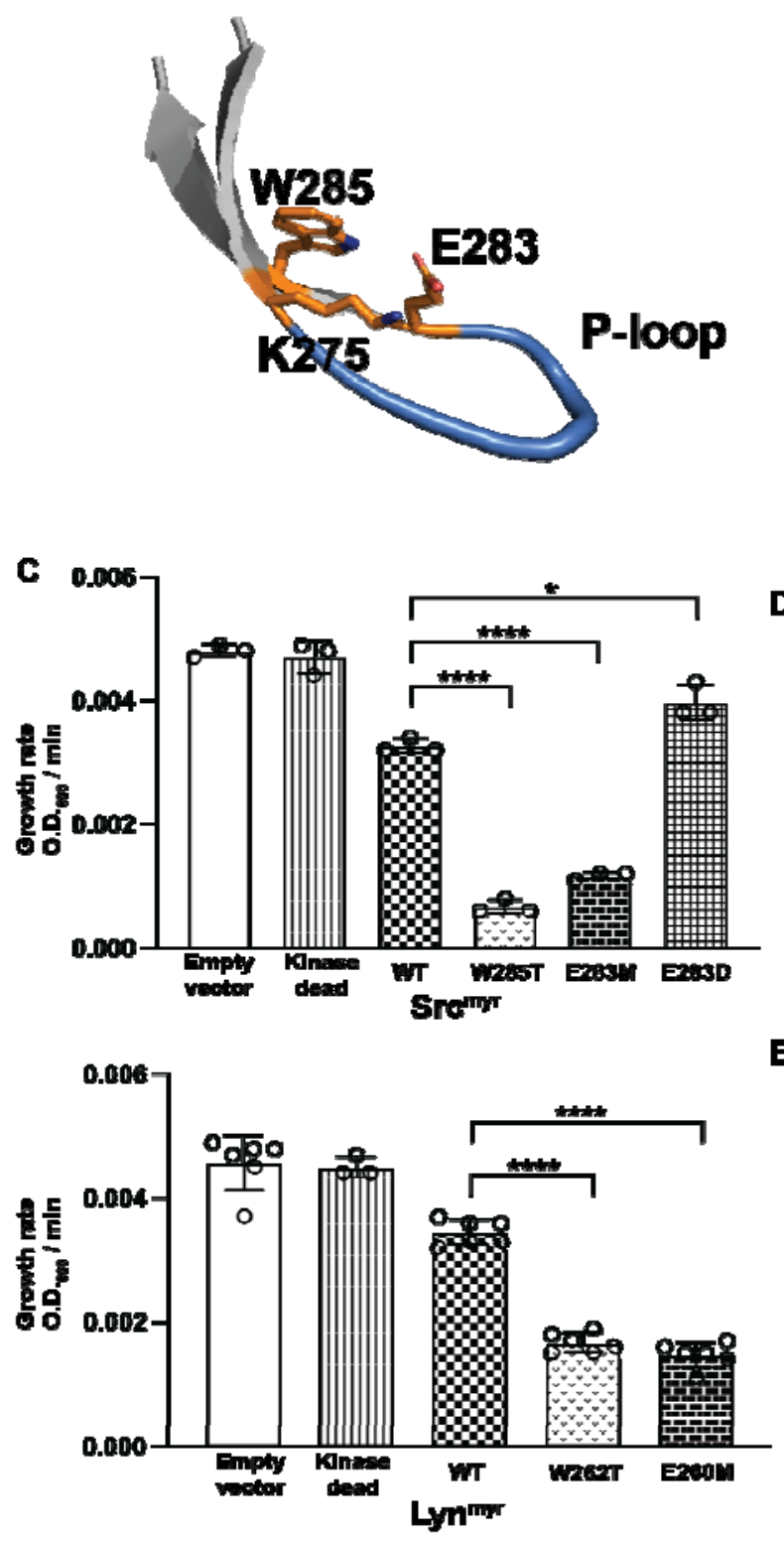

B
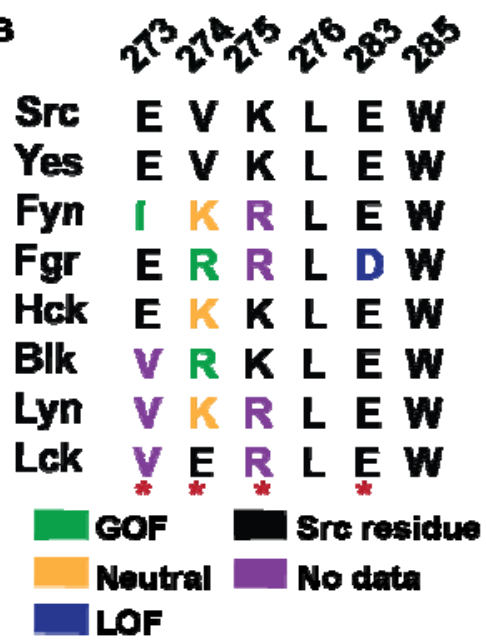

D
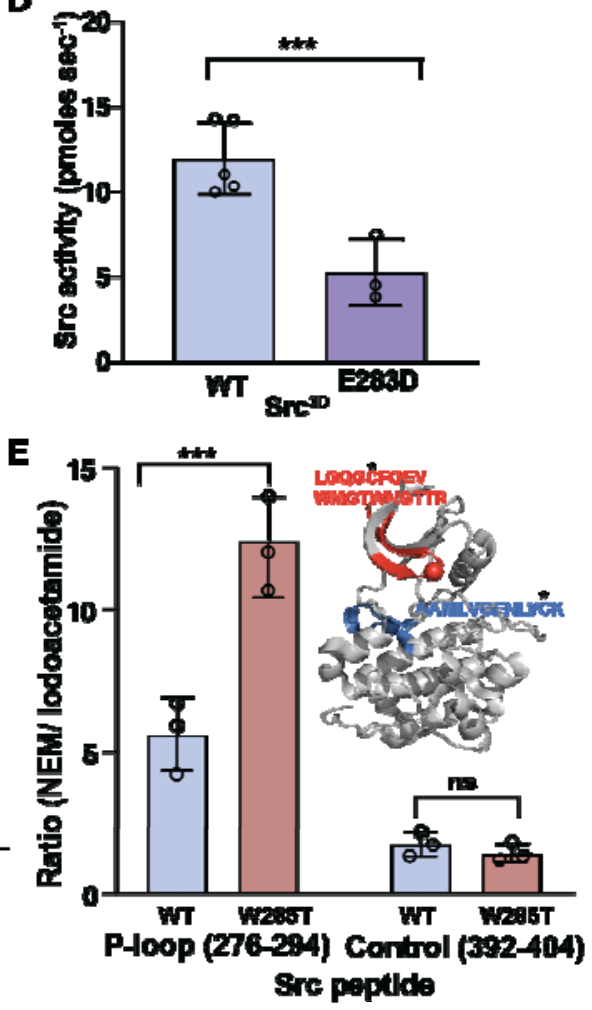

Figure 6. $\beta 1 / \beta 2$ resistance cluster mutations influence the dynamics of Src's P-loop. (A) Structure of the Nterminal lobe of Src's catalytic domain (PDB id: 1Y57) showing the flexible region of Src's P-loop in blue and K275 ( $\beta 1$ strand), E283 ( $\beta 2$ strand), and Trp285 ( $\beta 2$ strand) as orange sticks. (B) Sequence alignment of $\beta 1 / \beta 2$ resistance cluster residues in SFK. Classification of activating, WT-like and inactivating mutants derived from (Ahler et.al. 2019). (C) Growth rates of yeast expressing either (top) Src ${ }^{\text {myr }}$ (Kinase dead, WT, W285T, E283M, or E283D, $\mathrm{n}=3$ ) or (bottom) Lyn ${ }^{\text {myr }}$ (Kinase dead, WT, W262T, or E260M, $n=3-6$ ) (D) Phosphotransferase activity of purified WT and E283D $\operatorname{Src}^{\mathrm{CD}}\left(\mathrm{n}=3-5,{ }^{\star \star \star} P<0.001\right)$. (E) Peak intensity ratios of $\mathrm{N}$-ethylmaleimide (NEM) to iodoacetamide labeling of a Cys-containing tryptic peptide from Src's P-loop $\left(n=3,{ }^{* * *} P<0.001\right)$ and a Cys-containing control tryptic peptide in Src's C-terminal lobe $(n=3, n s=$ non-significant, $P>0.05)$ from purified WT and W285T SrC ${ }^{C D}$. The inset shows the structure of Src's catalytic domain (PDB id: 1Y57) with the Cys-containing P-loop peptide and its sequence shown in red and the Cys-containing control peptide and its sequence shown in blue. Labeled Cys residues are shown as spheres.

\section{DISCUSSION}


Here, we describe the first saturation mutagenesis study of drug resistance in a multi-domain protein kinaseSrc. We used a yeast-based assay that allowed us to compare how mutations affect Src's phosphotransferase activity in the absence of inhibitors with its activity in the presence of various ATP-competitive inhibitors. We observed that most inhibitor resistance mutations had a WT-like or activating effect on Src's phosphotransferase activity in the absence of inhibitors, which is consistent with the necessity of Src maintaining sufficient catalytic activity in order to obtain drug resistance. We also found that mutations at many residues that the line the ATP-binding site of Src were capable of conferring resistance to inhibition. Our results with conformation-selective, ATP-competitive inhibitors highlight that many of the ATP-binding site residues where these resistance mutations occur are inhibitor independent but that the conformational rearrangements required for certain modes of ATP-competitive inhibition raises opportunities for unique mechanisms of resistance to occur. Despite the large number of resistance mutations that line the ATP-binding site of Src, inhibitor contact residues did not represent the most general sites of resistance formation. Instead, residues that participate in the autoinhibition of Src were particularly prone to the development of resistance and mutations that both reduce inhibitor affinity and increase Src's phosphotransferase activity provided the strongest levels of resistance.

Numerous mutations in a spatially-defined cluster of six residues located in the N-terminal lobe of Src's catalytic, which we refer to as the $\beta 1 / \beta 2$ resistance cluster, were capable of conferring strong drug resistance. The ability of mutations within the $\beta 1 / \beta 2$ resistance cluster to both diminish inhibitor affinity and increase Src's phosphotransferase activity was surprising given its location on Src's catalytic domain. The side-chains of all but one (Leu276) of the six residues in $\beta 1 / \beta 2$ resistance cluster are directed away from Src's ATP-binding site and the top face of Src's N-terminal lobe has not previously been characterized as a regulatory interface. We found that residues within the $\beta 1 / \beta 2$ resistance cluster appear to be required for limiting the conformational dynamics of Src's N-terminal lobe and that mutations at these residues disrupt the autoinhibitory interactions required to downregulate Src's phosphotransferase activity. Furthermore, our biochemical analyses suggest that the $\beta 1 / \beta 2$ resistance cluster also modulates the dynamics of Src's P-loop. Residues within the P-loop of the tyrosine kinase Abl, which is closely-related to the SFKs, are major sites of resistance to inhibitors of the fusion protein BCR-Abl in the clinic (Figure S6C, D). (Redaelli et al., 2009; Seeliger et al., 2009) However, we observed very few inhibitor resistance mutations in Src's P-loop because almost all substitutions at these residues were found to be inactivating in our yeast growth assay (Figure S6E). Thus, the ability of activating $\beta 1 / \beta 2$ resistance cluster mutations to modulate the conformation of Src's P-loop while enhancing its phosphotransferase activity provides a potential route for acquiring drug resistance that does not require mutation of the P-loop itself. Together, our studies highlight the insights into kinase dynamics and regulation that can be obtained by performing systematic analysis of drug resistance.

\section{SIGNIFICANCE}

Proteins kinases have demonstrated a remarkable ability to acquire mutations that render them less susceptible to inhibition by ATP-competitive inhibitors. Despite the cataloguing of numerous resistance mutations with model studies and in the clinic, a comprehensive understanding of the mechanistic basis of kinase drug resistance is still lacking. To gain a greater understanding of drug resistance in a multi-domain protein kinase, we used a yeast-based screen to determine how almost every mutation in Src's catalytic domain affects its ability to be inhibited by ATP-competitive inhibitors. Using our yeast-based assay, we compared how mutations affect Src's phosphotransferase activity in the absence of inhibitors with its activity in the presence of various ATP-competitive inhibitors. As expected, we observed that most inhibitor resistance mutations had a WT-like or activating effect on Src's phosphotransferase activity in the absence of inhibitors. We also found that a number of mutations at positions that the line the ATP-binding site of Src provided general resistance to ATP-competitive inhibitors. However, a subset of resistance mutations appear to be specific to the structural rearrangements required for certain modes of ATP-competitive inhibition. While inhibitor contact residues represented sites of acquired resistance, we found that residues that participate in the autoinhibition of $\mathrm{Src}$ are particularly prone to the development of resistance. Biochemical analysis of a resistance-prone cluster of residues revealed that the top face of Src catalytic domain's N-terminal lobe, unexpectedly, contributes to the autoinhibition of $\mathrm{Src}$ and that mutations in this region led to resistance by lowering inhibitor affinity and promoting kinase hyperactivation. Together, our studies demonstrate how comprehensive profiling of drug resistance can be used to understand potential resistance pathways and uncover new mechanisms of kinase regulation. 


\section{REFERENCES}

(!!! INVALID CITATION !!! (data from Ahler et al., 2019)).

Agius, M.P., Ko, K.S., Johnson, T.K., Kwarcinski, F.E., Phadke, S., Lachacz, E.J., and Soellner, M.B. (2019). Selective Proteolysis to Study the Global Conformation and Regulatory Mechanisms of c-Src Kinase. ACS Chem Biol 14, 1556-1563. 10.1021/acschembio.9b00306.

Ahler, E., Register, A.C., Chakraborty, S., Fang, L., Dieter, E.M., Sitko, K.A., Vidadala, R.S.R., Trevillian, B.M., Golkowski, M., Gelman, H., et al. (2019). A Combined Approach Reveals a Regulatory Mechanism Coupling Src's Kinase Activity, Localization, and PhosphotransferaseIndependent Functions. Mol Cell 74, 393-408 e320. 10.1016/j.molcel.2019.02.003.

Azam, M., Seeliger, M.A., Gray, N.S., Kuriyan, J., and Daley, G.Q. (2008). Activation of tyrosine kinases by mutation of the gatekeeper threonine. Nat Struct Mol Biol 15, 1109-1118. $10.1038 /$ nsmb.1486.

Balzano, D., Santaguida, S., Musacchio, A., and Villa, F. (2011). A general framework for inhibitor resistance in protein kinases. Chem Biol 18, 966-975. 10.1016/j.chembiol.2011.04.013.

Barouch-Bentov, R., Che, J., Lee, C.C., Yang, Y., Herman, A., Jia, Y., Velentza, A., Watson, J., Sternberg, L., Kim, S., et al. (2009). A conserved salt bridge in the G loop of multiple protein kinases is important for catalysis and for in vivo Lyn function. Mol Cell 33, 43-52.

10.1016/j.molcel.2008.12.024.

Brugge, J.S., Jarosik, G., Andersen, J., Queral-Lustig, A., Fedor-Chaiken, M., and Broach, J.R. (1987). Expression of Rous sarcoma virus transforming protein pp60v-src in Saccharomyces cerevisiae cells. Mol Cell Biol 7, 2180-2187. 10.1128/mcb.7.6.2180-2187.1987.

Fang, L., Vilas-Boas, J., Chakraborty, S., Potter, Z.E., Register, A.C., Seeliger, M.A., and Maly, D.J. (2020). How ATP-Competitive Inhibitors Allosterically Modulate Tyrosine Kinases That Contain a Src-like Regulatory Architecture. ACS Chem Biol 15, 2005-2016. 10.1021/acschembio.0c00429.

Fowler, D.M., Araya, C.L., Fleishman, S.J., Kellogg, E.H., Stephany, J.J., Baker, D., and Fields, S. (2010). High-resolution mapping of protein sequence-function relationships. Nat Methods 7 ,

741-746. 10.1038/nmeth.1492.

Fowler, D.M., and Fields, S. (2014). Deep mutational scanning: a new style of protein science. Nat Methods 11, 801-807. 10.1038/nmeth.3027.

Garraway, L.A., and Janne, P.A. (2012). Circumventing cancer drug resistance in the era of personalized medicine. Cancer Discov 2, 214-226. 10.1158/2159-8290.CD-12-0012.

Gharwan, H., and Groninger, H. (2016). Kinase inhibitors and monoclonal antibodies in oncology: clinical implications. Nat Rev Clin Oncol 13, 209-227. 10.1038/nrclinonc.2015.213. Gibbons, D.L., Pricl, S., Kantarjian, H., Cortes, J., and Quintas-Cardama, A. (2012). The rise and fall of gatekeeper mutations? The BCR-ABL1 T315I paradigm. Cancer 118, 293-299. 10.1002/cncr.26225.

Krishnamurty, R., and Maly, D.J. (2010). Biochemical mechanisms of resistance to smallmolecule protein kinase inhibitors. ACS Chem Biol 5, 121-138. 10.1021/cb9002656.

Kritzer, J.A., Freyzon, Y., and Lindquist, S. (2018). Yeast can accommodate phosphotyrosine: vSrc toxicity in yeast arises from a single disrupted pathway. FEMS Yeast Res 18. 10.1093/femsyr/foy027. 
Lovly, C.M., and Shaw, A.T. (2014). Molecular pathways: resistance to kinase inhibitors and implications for therapeutic strategies. Clin Cancer Res 20, 2249-2256. 10.1158/1078-0432.CCR13-1610.

Persky, N.S., Hernandez, D., Do Carmo, M., Brenan, L., Cohen, O., Kitajima, S., Nayar, U., Walker, A., Pantel, S., Lee, Y., et al. (2020). Defining the landscape of ATP-competitive inhibitor resistance residues in protein kinases. Nat Struct Mol Biol 27, 92-104. 10.1038/s41594-0190358-z.

Ranjitkar, P., Brock, A.M., and Maly, D.J. (2010). Affinity reagents that target a specific inactive form of protein kinases. Chem Biol 17, 195-206. 10.1016/j.chembiol.2010.01.008.

Redaelli, S., Piazza, R., Rostagno, R., Magistroni, V., Perini, P., Marega, M., GambacortiPasserini, C., and Boschelli, F. (2009). Activity of bosutinib, dasatinib, and nilotinib against 18 imatinib-resistant BCR/ABL mutants. J Clin Oncol 27, 469-471. 10.1200/JCO.2008.19.8853.

S. Chakraborty, E.A., J. Simon, L. Fang, Z. E. Potter, K. A. Sitko, Jason J. Stephany, M. Guttman, D. M. Fowler, D. J. Maly (2021). Analysis of the drug resistance of thousands of Src tyrosine kinase mutants uncovers an unexpected regulatory network BioRxiv.

Seeliger, M.A., Ranjitkar, P., Kasap, C., Shan, Y., Shaw, D.E., Shah, N.P., Kuriyan, J., and Maly, D.J. (2009). Equally potent inhibition of $c-S r c$ and Abl by compounds that recognize inactive kinase conformations. Cancer Res 69, 2384-2392. 10.1158/0008-5472.CAN-08-3953.

Yun, C.H., Mengwasser, K.E., Toms, A.V., Woo, M.S., Greulich, H., Wong, K.K., Meyerson, M., and Eck, M.J. (2008). The T790M mutation in EGFR kinase causes drug resistance by increasing the affinity for ATP. Proc Natl Acad Sci U S A 105, 2070-2075. 10.1073/pnas.0709662105. Zhang, J., Yang, P.L., and Gray, N.S. (2009). Targeting cancer with small molecule kinase inhibitors. Nat Rev Cancer 9, 28-39. 10.1038/nrc2559. 